\title{
Solvability and coerciveness of multi-point Sturm-Liouville problems with abstract linear functionals
}

\author{
Yulin Bai ${ }^{1,2}$, Wanyi Wang ${ }^{1,3}$ and Kun $\mathrm{Li}^{4^{*}}$ (D)
}

"Correspondence: qslikun@163.com ${ }^{4}$ School of Mathematical Sciences, Qufu Normal University, Qufu, P.R. China

Full list of author information is available at the end of the article

\begin{abstract}
In this paper, we consider a Sturm-Liouville problem with finite discontinuous points inside an interval and with abstract linear functionals in the boundary and transmission conditions. For such a problem, the properties such as isomorphism, Fredholmness, and coerciveness with respect to the spectral parameter are investigated.
\end{abstract}

Keywords: Sturm-Liouville problems; Transmission conditions; Isomorphism; Coerciveness; Solvability

\section{Introduction}

We will discuss the following differential equation:

$$
M(\lambda) y:=-\left(p y^{\prime}\right)^{\prime}(x)+\lambda^{2} y(x)+T y(x)=f(x), \quad x \in I
$$

with nonclassical boundary conditions

$$
\begin{aligned}
M_{k} y:= & a_{01} y^{\left(n_{k}\right)}\left(\xi_{0}\right)+\sum_{h=1}^{n}\left(a_{h k} y^{\left(n_{k}\right)}\left(\xi_{h}-\right)+\tilde{a}_{h k} y^{\left(n_{k}\right)}\left(\xi_{h}+\right)\right) \\
& +a_{(n+1) k} y^{\left(n_{k}\right)}\left(\xi_{n+1}\right)+\sum_{j=1}^{n_{k}} \chi_{k j} y^{\left(n_{k}\right)}\left(x_{k j}\right)+F_{k} y=f_{k},
\end{aligned}
$$

where $k=1,2, \ldots, 2(n+1), \xi_{0}=-1, \xi_{h} \in(-1,1), \xi_{n+1}=1, \xi_{0}<\xi_{1}<\cdots<\xi_{n+1}$; set $I_{1}=\left[\xi_{0}, \xi_{1}\right)$, $I_{t}=\left(\xi_{t-1}, \xi_{t}\right), I_{n+1}=\left(\xi_{n}, \xi_{n+1}\right], I=\bigcup_{i=1}^{n+1} I_{i}$, and $J_{i}=\left(\xi_{i-1}, \xi_{i}\right), J=\bigcup_{i=1}^{n+1} J_{i}(h=1,2, \ldots, n ; t=$ $2,3, \ldots, n) ; p(x)$ is a piecewise constant function, $p(x)=p_{i}$ for $x \in I_{i}(i=1,2, \ldots, n+1)$; $T$ is a linear operator; $a_{0 k}, a_{h k}, \tilde{a}_{h k}, a_{(n+1) k}, \chi_{k j}, p_{i}\left(j=1,2, \ldots, n_{k}\right)$ are complex coefficients, and assume that $p_{i} \neq 0,\left|a_{0 k}\right|+\sum_{h=1}^{n}\left(\left|a_{h k}\right|+\left|\tilde{a}_{h k}\right|\right)+\left|a_{(n+1) k}\right| \neq 0$; $\lambda$ is the complex parameter; $n_{k}$ are integers; $x_{k j} \in J$ are internal points; $F_{k}$ is a linear function in the space $L_{q}[-1,1]$ $\left(L_{q}[-1,1]\right.$ is a set of $q$ th order integrable functions on $\left.[-1,1]\right)$.

In recent years, the classical Sturm-Liouville problem has been generalized into various types for its new importance in physical sciences and applied mathematics. For example, theoretical investigations have become focused on the discontinuous Sturm-Liouville

(c) The Author(s) 2019. This article is distributed under the terms of the Creative Commons Attribution 4.0 International License (http://creativecommons.org/licenses/by/4.0/), which permits unrestricted use, distribution, and reproduction in any medium, provided you give appropriate credit to the original author(s) and the source, provide a link to the Creative Commons license, and indicate if changes were made. 
problems for their application in physics. The discontinuity of the coefficients of the equations in the Sturm-Liouville problems corresponds to the fact that the heterogeneous media consist of two different materials. Moreover, boundary value problems with discontinuities arise in many physical problems such as heat and mass transfer, electrostatics, and diffraction problem [1,2]. It should be noted that some works on the spectral properties and coercive solvability of boundary value problems in Sobolev spaces can be found in $[3,4]$. Some boundary value problems for differential equations with discontinuous coefficients were investigated by Rasulov [5]. Note that an abstract theory of the boundary value problems with continuous coefficients and an eigenvalue parameter in the boundary conditions have been constructed by Yakubov and Yakubov (see [4] and corresponding bibliography). Many authors have been devoted to the study of discontinuous problems [6-22]. To deal with the discontinuity of the problem, transmission conditions are imposed on the discontinuous points. There are also other terminologies such as point interaction, interface condition, etc. [18, 23]. The properties of isomorphism, Fredholmness, and coerciveness of Sturm-Liouville problems with one discontinuous point were investigated by Mukhtarov and his coauthors in [6, 8, 9, 24].

In this paper, we investigate a Sturm-Liouville problem with discontinuities at finite points and with abstract linear functionals in the boundary-transmission conditions. We obtain the properties such as isomorphism, Fredholmness, and coerciveness of this problem.

\section{Boundary value problems with nonhomogeneous transmission conditions}

In this section, we consider the homogeneous differential equation

$$
M_{0}(\lambda) y:=-\left(p y^{\prime}\right)^{\prime}(x)+\lambda^{2} y(x)=0, \quad x \in I
$$

with the nonclassical boundary conditions

$$
M_{k 0} y:=a_{0 k} y^{\left(n_{k}\right)}\left(\xi_{0}\right)+\sum_{h=1}^{n}\left(a_{h k} y^{\left(n_{k}\right)}\left(\xi_{h}-\right)+\tilde{a}_{h k} y^{\left(n_{k}\right)}\left(\xi_{h}+\right)\right)+a_{(n+1) k} y^{\left(n_{k}\right)}\left(\xi_{n+1}\right)=f_{k},
$$

where $k=1,2, \ldots, 2(n+1)$. We shall use the notations

$$
\begin{aligned}
& \Gamma_{k} y:=\sum_{j=1}^{n_{k}} \chi_{k j} y^{\left(n_{k}\right)}\left(x_{k j}\right), \quad k=1,2, \ldots, 2(n+1), \\
& w_{2 i-1}:=p_{i}^{-1 / 2}, \quad w_{2 i}:=-p_{i}^{-1 / 2}, \quad i=1,2, \ldots, n+1, \\
& \alpha:=\min _{1 \leq i \leq n+1}\left\{\arg p_{i}\right\}, \quad \beta:=\max _{1 \leq i \leq n+1}\left\{\arg p_{i}\right\}, \\
& \omega=\left[\begin{array}{ccccc}
a_{01} w_{1}^{n_{1}} & a_{11} w_{2}^{n_{1}} & \tilde{a}_{11} w_{3}^{n_{1}} & \ldots & a_{(n+1) 1} w_{2(n+1)}^{n_{1}} \\
a_{02} w_{1}^{n_{2}} & a_{12} w_{2}^{n_{2}} & \tilde{a}_{12} w_{3}^{n_{2}} & \ldots & a_{(n+1) 2} w_{2(n+1)}^{n_{2}} \\
a_{03} w_{1}^{n_{3}} & a_{13} w_{2}^{n_{3}} & \tilde{a}_{13} w_{3}^{n_{3}} & \ldots & a_{(n+1) 3} w_{2(n+1)}^{n_{3}} \\
\vdots & \vdots & \vdots & \vdots & \vdots \\
a_{0,2(n+1)} w_{1}^{n_{2(n+1)}} & a_{1,2(n+1)} w_{2}^{n_{2(n+1)}} & \tilde{a}_{1,2(n+1)} w_{3}^{n_{2(n+1)}} & \cdots & a_{(n+1), 2(n+1)} w_{2(n+1)}^{n_{2(n+1)}}
\end{array}\right]
\end{aligned}
$$


and

$$
\Omega_{\varepsilon}(\alpha, \beta):=\left\{\lambda \in \mathbb{C} \mid \frac{1}{2}(\pi+\beta+\varepsilon)<\arg \lambda<\frac{1}{2}(3 \pi+\alpha-\varepsilon)\right\}
$$

for real $\varepsilon>0$ sufficiently small.

Note that the direct sum of Sobolev spaces $W_{q}^{m}=W_{q}^{m}\left(J_{1}\right) \oplus W_{q}^{m}\left(J_{2}\right) \oplus \cdots \oplus W_{q}^{m}\left(J_{n}\right)$ (for an integer $m \geq 0$ and real $q>1$ ) is defined as a Banach space of complex-valued function $y=y(x)$ on $I$ which belongs to $W_{q}^{m}\left(J_{i}\right)(i=1,2, \ldots,(n+1))$ in intervals $J_{i}$ respectively, with the norm

$$
\|y\|_{q, m}:=\sum_{i=1}^{n+1}\|y\|_{W_{q}^{m}\left(J_{i}\right)}
$$

Here, as usual, $W_{q}^{m}(a, b)$ is the Sobolev space, i.e., the Banach space consisting of all measurable functions that have generalized derivatives up to $m$ th order in the interval $(a, b)$ inclusive with the finite norm

$$
\|y\|_{W_{q}^{m}(a, b)}:=\sum_{\nu=0}^{m}\left(\int_{a}^{b}\left|y^{(v)}(x)\right|^{q} d x\right)^{1 / q}
$$

Theorem 2.1 If $\omega \neq 0$, then for any $\varepsilon>0$ there exists $\mu_{\varepsilon}>0$ such that, for all $\lambda \in \Omega_{\varepsilon}(\alpha, \beta)$ for which $|\lambda|>\mu_{\varepsilon}$, problem (2.1)-(2.2) has a unique solution $y(x, \lambda) \in W_{q}^{\sigma}$ for arbitrary $\sigma \geq \max \left\{2, \max _{1 \leq k \leq 2(n+1)}\left\{n_{k}\right\}+1\right\}$ and $\lambda$ has the following coercive estimate:

$$
\sum_{\ell=1}^{\sigma}\|y\|_{q, \ell} \leq C(\varepsilon) \sum_{k=1}^{2(n+1)}|\lambda|^{\sigma-n_{k}-q^{-1}}\left|f_{k}\right| .
$$

Proof Let $y_{k}(x, \lambda)(k=1,2, \ldots, 2(n+1))$ be the basic solution of equation $(2.1)$, then $y_{k}(x, \lambda)$ can be represented as

$$
y_{k}(x, \lambda)= \begin{cases}\exp \left(w_{k} \lambda\left(x-\tilde{\xi}_{k}\right)\right), & \text { for } x \in \tilde{I}_{k} ; \\ 0, & \text { for } x \notin \tilde{I}_{k},\end{cases}
$$

where $\tilde{I}_{2 i-1}=\tilde{I}_{2 i}=I_{i}$ for every $i, k=2 i-1,2 i(i=1,2, \ldots, n+1), \tilde{\xi}_{1}=\xi_{0}, \tilde{\xi}_{2 h}=\tilde{\xi}_{2 h+1}=\xi_{i}$, $\tilde{\xi}_{2(n+1)}=\xi_{n+1}(h=1,2, \ldots, n)$. It is clear that the general solution of $(2.1)$ can be written as

$$
y(x, \lambda)=\sum_{k=1}^{2(n+1)} C_{k} y_{k}(x, \lambda) .
$$

Substituting equation (2.5) into boundary-transmission conditions (2.2), we obtain a linear system with respect to $C_{k}(k=1,2, \ldots, 2(n+1))$

$$
\begin{aligned}
& \left(w_{1} \lambda\right)^{n_{k}}\left(a_{0 k}+a_{1 k} e^{w_{1} \lambda\left(\xi_{1}-\xi_{0}\right)}\right) C_{1}+\left(w_{2} \lambda\right)^{n_{k}}\left(a_{1 k}+a_{0 k} e^{w_{2} \lambda\left(\xi_{0}-\xi_{1}\right)}\right) C_{2} \\
& +\sum_{t=2}^{n+1}\left[\left(w_{2 t-1} \lambda\right)^{n_{k}}\left(\tilde{a}_{(t-1) k}+a_{t k} e^{w_{2 t-1} \lambda\left(\xi_{t}-\xi_{t-1}\right)}\right) C_{2 t-1}\right. \\
& \left.+\left(w_{2 t} \lambda\right)^{n_{k}}\left(a_{t k}+\tilde{a}_{(t-1) k} e^{w_{2 t} \lambda\left(\xi_{t-1}-\xi_{t}\right)}\right) C_{2 t}\right]=f_{k},
\end{aligned}
$$


where $k=1,2, \ldots, 2(n+1)$. It follows from $\lambda \in \Omega_{\varepsilon}(\alpha, \beta)$ that

$$
\begin{array}{ll}
\frac{1}{2}(\pi+\varepsilon)<\arg \left(w_{k} \lambda\right)<\frac{1}{2}(3 \pi-\varepsilon), & k=1,3, \ldots, 2 n+1 ; \\
-\frac{1}{2}(\pi-\varepsilon)<\arg \left(w_{k} \lambda\right)<\frac{1}{2}(\pi-\varepsilon), & k=2,4, \ldots, 2(n+1) .
\end{array}
$$

Therefore, for this $\lambda$ and $\varepsilon>0$ (sufficiently small), we obtain

$$
(-1)^{k+1} \operatorname{Re}\left(w_{k} \lambda\right)<-|\lambda|\left|w_{k}\right| \sin \frac{\varepsilon}{2}, \quad k=1,2, \ldots, 2(n+1) .
$$

Thus, the determinant of system (2.6) has the form

$$
A(\lambda)=\lambda^{\tilde{n}}(\omega+\theta(\lambda))
$$

where $\tilde{n}=n_{1}+n_{2}+\cdots+n_{2(n+1)}$,

$$
\theta(\lambda)=e^{\kappa}\left[\begin{array}{cccc}
a_{11} w_{1}^{n_{1}} & a_{01} w_{2}^{n_{1}} & \cdots & \tilde{a}_{n 1} w_{2(n+1)}^{n_{1}} \\
a_{12} w_{1}^{n_{2}} & a_{02} w_{2}^{n_{2}} & \cdots & \tilde{a}_{n 2} w_{2(n+1)}^{n_{2}} \\
\vdots & \vdots & \vdots & \vdots \\
a_{1,2(n+1)} w_{1}^{n_{2(n+1)}} & a_{0,2(n+1)} w_{2}^{n_{2(n+1)}} & \cdots & \tilde{a}_{n, 2(n+1)} w_{2(n+1)}^{n_{2(n+1)}}
\end{array}\right],
$$

$\kappa=\lambda \sum_{i=1}^{n+1}\left(\xi_{i}-\xi_{i-1}\right)\left(w_{2 i-1}-w_{2 i}\right)$ and $\theta(\lambda) \rightarrow 0$ as $|\lambda| \rightarrow \infty$ in the angle $\Omega_{\varepsilon}(\alpha, \beta)$. Since $\omega \neq 0$, there exists $\mu_{\varepsilon}>0$ such that, for all $\lambda \in \Omega_{\varepsilon}(\alpha, \beta)$ and $|\lambda|>\mu_{\varepsilon}$, we have $A(\lambda) \neq 0$. So, for these $\lambda$, the unique solution for the system of linear homogeneous equations (2.6) has the following representation:

$$
C_{k}(\lambda)=\frac{1}{A(\lambda)} \sum_{\eta=1}^{2(n+1)} A_{\eta k}(\lambda) f_{\eta}, \quad k=1,2, \ldots, 2(n+1)
$$

where $A_{\eta k}(\lambda)$ is an algebraic cofactor of $(\eta, k)$ th element of the determinant $A(\lambda)$. It is obvious that each of the determinants $A_{\eta k}(\lambda)$ can be represented as

$$
A_{\eta k}(\lambda)=\lambda^{\tilde{n}-n_{\eta}}\left(\omega_{\eta k}+\theta_{\eta k}(\lambda)\right)
$$

where $\omega_{\eta k} \in \mathbb{C}$ and $\theta(\lambda) \rightarrow 0$ as $|\lambda| \rightarrow \infty$ in the angle $\Omega_{\varepsilon}(\alpha, \beta)$. Hence, we have

$$
C_{k}(\lambda)=\sum_{\eta=1}^{2(n+1)} \lambda^{-n_{\eta}} \frac{\omega_{\eta k}+\theta_{\eta k}(\lambda)}{\omega+\theta(\lambda)} f_{\eta}, \quad k=1,2, \ldots, 2(n+1)
$$

So, the solution of (2.1)-(2.2) has the following representation:

$$
y(x, \lambda)=\sum_{k=1}^{2(n+1)} \sum_{\eta=1}^{2(n+1)} \lambda^{-n_{\eta}} \frac{\omega_{\eta k}+\theta_{\eta k}(\lambda)}{\omega+\theta(\lambda)} f_{\eta} y_{k}(x, \lambda) .
$$


From the expression of $y(x, \lambda)$ we obtain that, for each integer $\tau \geq 0$ and $\lambda \in \Omega_{\varepsilon}(\alpha, \beta)$, $|\lambda| \rightarrow \infty$, the estimate

$$
\left\|y^{(\tau)}\right\|_{L_{q}(-1,1)} \leq C \sum_{\eta=1}^{2(n+1)}\left(|\lambda|^{\tau-n_{\eta}}\left|f_{\eta}\right| \sum_{k=1}^{2(n+1)}\left\|y_{k}(x, \lambda)\right\|_{L q\left(\tilde{I}_{k}\right)}\right)
$$

is valid. Further, the inequalities

$$
\begin{aligned}
\left\|y_{2 i-1}(x, \lambda)\right\|_{L_{q}\left(\tilde{I}_{2 i-1}\right)}^{q} & =\int_{\xi_{i-1}}^{\xi_{i}} e^{q \operatorname{Re}\left(w_{2 i-1} \lambda\right)\left(x-\xi_{i-1}\right)} d x \\
& \leq \int_{\xi_{i-1}}^{\xi_{i}} e^{-q\left|w_{2 i-1}\right||\lambda|\left(x-\xi_{i-1}\right) \sin \frac{\varepsilon}{2}} d x \\
& ={ }^{t=|\lambda|\left(x-\xi_{i-1}\right)} \int_{0}^{|\lambda|\left(\xi_{i}-\xi_{i-1}\right)} e^{-q\left|w_{2 i-1}\right|\left(\sin \frac{\varepsilon}{2}\right) t} d\left(\frac{t}{|\lambda|}+\xi_{i-1}\right) \\
& \leq|\lambda|^{-1} \int_{0}^{+\infty} e^{-q\left|w_{2 i-1}\right|\left(\sin \frac{\varepsilon}{2}\right) t} d t \\
& =|\lambda|^{-1}\left(q\left|w_{2 i-1}\right| \sin \frac{\varepsilon}{2}\right)^{-1} \\
& =C_{2 i-1}(\varepsilon)|\lambda|^{-1}, \\
\left\|y_{2 i}(x, \lambda)\right\|_{L_{q}\left(\tilde{I}_{2 i}\right)}^{q}= & \int_{\xi_{i-1}}^{\xi_{i}} e^{q \operatorname{Re}\left(w_{2 i} \lambda\right)\left(x-\xi_{i}\right)} d x \\
\leq & \int_{\xi_{i-1}}^{\xi_{i}} e^{-q\left|w_{2 i}\right||\lambda|\left(\xi_{i}-x\right) \sin \frac{\varepsilon}{2}} d x \\
= & { }^{t|\lambda|\left(\xi_{i}-x\right)} \int_{|\lambda|\left(\xi_{i}-\xi_{i-1}\right)}^{0} e^{-q\left|w_{2 i}\right|\left(\sin \frac{\varepsilon}{2}\right) t} d\left(\xi_{i}-\frac{t}{|\lambda|}\right) \\
\leq & |\lambda|^{-1} \int_{0}^{+\infty} e^{-q\left|w_{2 i}\right|\left(\sin \frac{\varepsilon}{2}\right) t} d t \\
= & |\lambda|^{-1}\left(q\left|w_{2 i}\right| \sin \frac{\varepsilon}{2}\right)^{-1} \\
= & C_{2 i}(\varepsilon)|\lambda|^{-1},
\end{aligned}
$$

where $i=1,2, \ldots,(n+1)$, hold by (2.4). Substituting (2.8), (2.9) into (2.7) yields

$$
\left\|y^{(\tau)}(x)\right\|_{L_{q}(-1,1)} \leq C(\varepsilon) \sum_{\eta=1}^{2(n+1)}|\lambda|^{\tau-n_{\eta}-q^{-1}}\left|f_{\eta}\right|,
$$

which in turn gives us the needed estimation (2.3). The proof is completed.

\section{Fredholm property for multi-point boundary value problem with functional} conditions

Let $\widetilde{M}$ be the linear operator corresponding to problem (1.1)-(1.2). Suppose that $\sigma \geq$ $\max \left\{2, \max _{1 \leq k \leq 2(n+1)}\left\{n_{k}\right\}+1\right\}$ and define $\widetilde{M}$ from $W_{q}^{\sigma}$ into $W_{q}^{\sigma-2} \oplus \mathbb{C}^{2(n+1)}$ by the rule

$$
\tilde{M} y=\left(M(\lambda), M_{1} y, M_{2} y, \ldots, M_{2(n+1)} y\right) .
$$


Theorem 3.1 Assume that the following conditions hold:

(1) For $x \in I_{i}, p_{i} \neq 0$;

(2) $F_{k}(k=1,2, \ldots, 2(n+1))$ are continuous functionals in $W_{q}^{\sigma}$;

(3) The operator $T$ from $W_{q}^{\sigma}$ into $W_{q}^{\sigma-2}$ is compact.

Then $\widetilde{M}$ is bounded and Fredholm operator.

Proof The operator $\tilde{M}$ can be represented as

$$
\begin{aligned}
& \widetilde{M}_{0} y=\left(M_{0}(\lambda) y, M_{10} y, M_{20} y, \ldots, M_{2(n+1) 0} y\right), \\
& \tilde{M}_{1} y=\left(T y, \Gamma_{1} y+F_{1} y, \Gamma_{2} y+F_{2} y, \ldots, \Gamma_{2(n+1)} y+F_{2(n+1)} y\right) .
\end{aligned}
$$

The operator $\widetilde{M}_{0}$ is an isomorphism from $W_{q}^{\sigma}$ onto $W_{q}^{\sigma-2} \oplus \mathbb{C}^{2(n+1)}$ by Theorem 2.1. Furthermore, it follows from (2) and (3) that the operator $\widetilde{M}_{1}$ acts compactly from $W_{q}^{\sigma}$ onto $W_{q}^{\sigma-2} \oplus \mathbb{C}^{2(n+1)}$.

Therefore, by the definition of isomorphism and Theorem 1.2.8 in [3] (or [25, p. 238]), the operator $\widetilde{M}=\widetilde{M}_{0}+\widetilde{M}_{1}$ is Fredholm. Moreover, it is obvious that the operator $\widetilde{M}$ is bounded. So, the desired results are obtained.

\section{Isomorphism and coerciveness of the principal part of the problem}

We consider the principle part of main problem (1.1)-(1.2) without internal points, that is,

$$
\begin{aligned}
M_{0}(\lambda) y:= & -\left(p y^{\prime}\right)^{\prime}(x)+\lambda^{2} y(x)=f(x), \quad x \in I, \\
M_{k 0} y:= & a_{0 k} y^{\left(n_{k}\right)}\left(\xi_{0}\right)+\sum_{h=1}^{n}\left(a_{h k} y^{\left(n_{k}\right)}\left(\xi_{h}-\right)+\tilde{a}_{h k} y^{\left(n_{k}\right)}\left(\xi_{h}+\right)\right) \\
& +a_{(n+1) k} y^{\left(n_{k}\right)}\left(\xi_{n+1}\right)=f_{k}
\end{aligned}
$$

for $k=1,2, \ldots, 2(n+1)$. The corresponding operator is

$$
\widehat{M}_{0} y:=\left(M_{0}(\lambda) y, M_{10} y, M_{20} y, \ldots, M_{2(n+1) 0} y\right) .
$$

Theorem 4.1 Let the condition $\omega \neq 0$ and $\sigma \geq \max \left\{2, \max _{1 \leq k \leq 2(n+1)}\left\{n_{k}\right\}+1\right\}$ be satisfied. Then, for each $\varepsilon>0$, there exists $\mu_{\varepsilon}>0$ such that, for all complex numbers $\lambda \in \Omega_{\varepsilon}(\alpha, \beta)$, $|\lambda|>\mu_{\varepsilon}$, the operator $\widehat{M}_{0}$ from $W_{q}^{\sigma}$ onto $W_{q}^{\sigma-2} \oplus \mathbb{C}^{2(n+1)}$ is an isomorphism, and for these $\lambda$ the inequality

$$
\sum_{\ell=0}^{\sigma}|\lambda|^{\sigma-\ell}\|y\|_{q, \ell} \leq C(\varepsilon)\left(\|f\|_{q, \sigma-2}+|\lambda|^{\sigma-2}\|f\|_{q, 0}+\sum_{k=1}^{2(n+1)}|\lambda|^{\sigma-n_{k}-q^{-1}}\left|f_{k}\right|\right)
$$

holds for the solution of (4.1)-(4.2).

Proof Obviously, the linear operator $\widehat{M}_{0}$ acts continuously from the space $W_{q}^{\sigma}$ into $W_{q}^{\sigma-2} \oplus \mathbb{C}^{2(n+1)}$. Let us prove that, for any $\left(f(x), f_{1}, f_{2}, \ldots, f_{2(n+1)}\right) \in W_{q}^{\sigma-2} \oplus \mathbb{C}^{2(n+1)}$ and $f_{i}$, problem (4.1)-(4.2) has a unique solution belonging to $W_{q}^{\sigma}$. Denote by $f_{i}(x)$ the restriction of $f(x)$ on the interval $J_{i}$. Let $\tilde{f}_{i}(x) \in W_{q}^{\sigma-2}(\mathbb{R})$ be an extension of $f_{i}(x) \in W_{q}^{\sigma-2}\left(J_{i}\right)$. By 
Lemma 1.7.6 in [3] there exists an extension operator $T_{i} f_{i}:=\tilde{f}_{i}$ from $W_{q}^{\sigma-2}$ into $W_{q}^{\sigma-2}(\mathbb{R})$ is bounded for $i=1,2, \ldots, n+1$, where as usual $\mathbb{R}=(-\infty,+\infty)$. We shall find the solution $y(x, \lambda)$ of problem (4.1)-(4.2) in the form of $y(x, \lambda)=y_{1}(x, \lambda)+y_{2}(x, \lambda)$, where $y_{1}(x, \lambda)=$ $\left(y_{1 i}(x, \lambda)\right)$, the function $y_{1 i}(x, \lambda)$ is the restriction of the solution $\tilde{y}_{1 i}(x, \lambda)$ on $J_{i}$ of the following equation:

$$
-\left(p_{i} \tilde{y}^{\prime}\right)^{\prime}(x)+\lambda^{2} \tilde{y}(x)=\tilde{f}_{i}(x), \quad x \in \mathbb{R}
$$

for $i=1,2, \ldots, n+1$.

By virtue of Theorem 3.2.1 in [3], we get that this equation has a unique solution $\tilde{y}_{1 i}=$ $\tilde{y}_{1 i}(x, \lambda) \in W_{q}^{\sigma}(\mathbb{R})$, and for $y_{1 i}$, the estimate

$$
\sum_{\ell=0}^{\sigma}|\lambda|^{\sigma-\ell}\left\|y_{1 i}\right\|_{W_{q}^{\ell}\left(J_{i}\right)} \leq C(\varepsilon)\left(\|f\|_{W_{q}^{\sigma-2}\left(J_{i}\right)}+|\lambda|^{\sigma-2}\|f\|_{\left.L_{q} J_{i}\right)}\right)
$$

where $i=1,2, \ldots, n+1$, holds for all $\lambda \in \Omega_{\varepsilon}(\alpha, \beta)$ sufficiently large in modulus.

Hence, the function

$$
y_{1}(x, \lambda)=\left\{\begin{array}{cc}
y_{11}(x, \lambda), & \text { for } x \in J_{1} \\
y_{12}(x, \lambda), & \text { for } x \in J_{2} \\
\vdots & \\
y_{1, n+1}(x, \lambda), & \text { for } x \in J_{n+1}
\end{array}\right.
$$

satisfies equation (4.1), and from (4.4) the following estimate

$$
\sum_{\ell=0}^{\sigma}|\lambda|^{\sigma-\ell}\left\|y_{1}\right\|_{q, \ell} \leq C(\varepsilon)\left(\|f\|_{q, \sigma-2}+|\lambda|^{\sigma-2}\|f\|_{q, 0}\right)
$$

holds for all $\lambda \in \Omega_{\varepsilon}(\alpha, \beta)$ sufficiently large in modulus. In light of solution (4.5), consider the following boundary value problem:

$$
\begin{aligned}
& -\left(p y^{\prime}\right)^{\prime}(x)-\lambda^{2} y(x)=0, \quad x \in J, \\
& M_{k 0} y=f_{k}-M_{k 0} y_{1}(x, \lambda), \quad k=1,2, \ldots, 2(n+1) .
\end{aligned}
$$

By Theorem 2.1, this problem has a unique solution $y_{2}=y_{2}(x, \lambda) \in W_{q}^{\sigma}$ for all complex numbers $\lambda \in \Omega_{\varepsilon}(\alpha, \beta)$ sufficiently large in modulus, and for these $\lambda$ the estimate

$$
\sum_{\ell=0}^{\sigma}|\lambda|^{\sigma-\ell}\left\|y_{2}\right\|_{q, \ell} \leq C(\varepsilon) \sum_{k=1}^{2(n+1)}|\lambda|^{\sigma-n_{k}-q^{-1}}\left(\left|f_{k}\right|+\left|M_{k 0} y_{1}\right|\right)
$$

is valid. Applying Theorem 1.7.7/2 in [3] and (2.3), one has that, for all $\lambda \in \Omega_{\varepsilon}(\alpha, \beta)$ and $\sigma \geq \max \left\{2, \max _{1 \leq k \leq 2(n+1)}\left\{n_{k}\right\}+1\right\}$, the following estimates hold:

$$
\begin{aligned}
|\lambda|^{\sigma-n_{k}-q^{-1}}\left|M_{k 0} y_{1}\right| & \leq C|\lambda|^{\sigma-n_{k}-q^{-1}} \sum_{i=1}^{n+1}\left\|y_{1}\right\|_{C^{n} k\left(I_{i}\right)} \\
& \leq C \sum_{i=1}^{n+1}\left(|\lambda|^{\sigma}\left\|y_{1 i}\right\|_{L_{q}\left(J_{i}\right)}+\left\|y_{1 i}\right\|_{\left.W_{q}^{\sigma} J_{i}\right)}\right)
\end{aligned}
$$




$$
\begin{aligned}
& \leq C\left(|\lambda|^{\sigma}\left\|y_{1}\right\|_{q, 0}+\left\|y_{1}\right\|_{q, \sigma}\right) \\
& \leq C(\varepsilon)\left(|\lambda|_{\sigma-2}\|f\|_{q, 0}+\|f\|_{q, \sigma-2}\right) .
\end{aligned}
$$

Via (4.7) and (4.8) we have the inequality

$$
\sum_{\ell=0}^{\sigma}|\lambda|^{\sigma-\ell}\left\|y_{2}\right\|_{q, \ell} \leq C(\varepsilon)\left(\|f\|_{q, \sigma-2}+|\lambda|^{\sigma-2}\|f\|_{q, 0}+\sum_{k=1}^{2(n+1)}|\lambda|^{\sigma-n_{k}-q^{-1}}\left|f_{k}\right|\right) .
$$

It is easy to see that $y(x, \lambda)=y_{1}(x, \lambda)+y_{2}(x, \lambda)$ is the solution of problem (4.1)-(4.2). Taking into account estimates (4.6) and (4.9), we see that for this solution the needed estimation (4.3) is valid. Moreover, from estimate (4.3) the uniqueness of the solution follows. On the other hand, by Theorem 3.1 the operator $\widehat{M}_{0}$ is Fredholm from $W_{q}^{\sigma}$ onto $W_{q}^{\sigma-2} \oplus \mathbb{C}^{2(n+1)}$. Now, an isomorphism of this operator follows from the fact that it is a Fredholm and one-to-one operator. So, the proof of the theorem is completed.

\section{Solvability and coerciveness of the main problem with nonclassical boundary conditions}

Now, we will study the main problem (1.1)-(1.2).

Theorem 5.1 Assume that the following conditions hold:

(1) $\omega \neq 0$ and $\sigma \geq \max \left\{2, \max _{1 \leq k \leq 2(n+1)}\left\{n_{k}\right\}+1\right\}$;

(2) The operator $T$ from $W_{q}^{\sigma}$ into $W_{q}^{\sigma-2}$ is compact, and for all $\varepsilon>0$

$$
\begin{aligned}
& \|T y\|_{q, 0} \leq \varepsilon\|y\|_{q, 2}+C(\varepsilon)\|y\|_{q, 0}, \quad y \in W_{q}^{2} ; \\
& \|T y\|_{q, \sigma-2} \leq \varepsilon\|y\|_{q, \sigma}+C(\varepsilon)\|y\|_{q, 0}, \quad y \in W_{q}^{\sigma} ;
\end{aligned}
$$

(3) Functionals $F_{k}$ in $W_{q}^{n_{k}}(k=1,2, \ldots, 2(n+1))$ are continuous.

Then, for each $\varepsilon>0$, there exists $\mu_{\varepsilon}>0$ such that, for all $\lambda \in \Omega_{\varepsilon}(\alpha, \beta)$ and $|\lambda|>\mu_{\varepsilon}$, the operator

$$
\widehat{M} y=\left(M(\lambda) y, M_{1} y, M_{2} y, \ldots, M_{2(n+1)} y\right)
$$

is an isomorphism from $W_{q}^{\sigma}$ onto $W_{q}^{\sigma-2} \oplus \mathbb{C}^{2(n+1)}$, and for these $\lambda$ we have the following coercive estimate for the solution of problem (1.1)-(1.2):

$$
\sum_{\ell=0}^{\sigma}|\lambda|^{\sigma-\ell}\|y\|_{q, \ell} \leq C(\varepsilon)\left(\|f\|_{q, \sigma-2}+|\lambda|^{\sigma-2}\|f\|_{q, 0}+\sum_{k=1}^{2(n+1)}|\lambda|^{\sigma-n_{k}-q^{-1}}\left|f_{k}\right|\right),
$$

where $C(\varepsilon)$ is a constant which depends only on $\varepsilon$.

Proof Let $\left(f(x), f_{1}, f_{2}, \ldots, f_{2(n+1)}\right)$ be any element of $W_{q}^{\sigma-2} \oplus \mathbb{C}^{2(n+1)}$. Suppose that there exists a solution $y=y(x, \lambda)$ of problem (1.1)-(1.2) corresponding to this element. Then this solution satisfies the equalities

$$
M_{0}(\lambda) y:=-\left(p y^{\prime}\right)^{\prime}+\lambda^{2} y=M(\lambda) y-T y
$$




$$
\begin{aligned}
M_{k 0} y:= & a_{0 k} y^{\left(n_{k}\right)}\left(\xi_{0}\right)+\sum_{h=1}^{n}\left(a_{h k} y^{n_{k}}\left(\xi_{h}-\right)+\tilde{a}_{h k} y^{\left(n_{k}\right)}\left(\xi_{h}+\right)\right) \\
& +a_{(n+1) k} y^{\left(n_{k}\right)}\left(\xi_{n+1}\right)=M_{k} y-\Gamma_{k} y-F_{k} y
\end{aligned}
$$

where $k=1,2, \ldots, 2(n+1)$. By Theorem 4.1 we have that for this solution the following a priori estimates hold:

$$
\begin{aligned}
\sum_{\ell=0}^{\sigma}|\lambda|^{\sigma-\ell}\|y\|_{q, \ell} \leq & C(\varepsilon)\left(\|f\|_{q, \sigma-2}+|\lambda|^{\sigma-2}\|f\|_{q, 0}+\sum_{k=1}^{2(n+1)}|\lambda|^{\sigma-n_{k}-q^{-1}}\left|f_{k}\right|\right) \\
= & C(\varepsilon)\left(\|M(\lambda) y-T y\|_{q, \sigma-2}+|\lambda|^{\sigma-2}\|M(\lambda) y-T y\|_{q, 0}\right. \\
& \left.+\sum_{k=1}^{2(n+1)}|\lambda|^{\sigma-n_{k}-q^{-1}}\left|M_{k} y-\Gamma_{k} y-F_{k} y\right|\right) \\
\leq & C(\varepsilon)\left(\|f\|_{q, \sigma-2}+|\lambda|^{\sigma-2}\|f\|_{q, 0}+\|T y\|_{q, \sigma-2}+|\lambda|^{\sigma-2}\|T y\|_{q, 0}\right. \\
& \left.+\sum_{k=1}^{2(n+1)}|\lambda|^{\sigma-n_{k}-q^{-1}}\left(\left|f_{k}\right|+\left|\Gamma_{k} y\right|+\left|F_{k} y\right|\right)\right) .
\end{aligned}
$$

Let $\zeta$ be any real number satisfying

$$
\begin{aligned}
0 & <\zeta \\
& <\min \left\{\frac{\xi_{i}-\xi_{i-1}}{2},\left|\xi_{i}-x_{k j}\right|,\left|\xi_{0}-x_{k j}\right|:\right. \\
& \left.\quad i=1,2, \ldots, n+1 ; k=1,2, \ldots, 2(n+1) ; j=1,2, \ldots, n_{k}\right\} .
\end{aligned}
$$

Using the same method in [15, Sect. 2.8.3], it is not difficult to construct a function $\phi(x) \in$ $\mathbb{C}_{0}^{\infty}(\mathbb{R})$ such that

$$
\phi(x)= \begin{cases}1, & x \in \bigcup_{i=1}^{n+1}\left[\xi_{i-1}+\zeta, \xi_{i}-\zeta\right] \\ 0, & x \in \bigcup_{i=1}^{n+1}\left[\xi_{0}, \xi_{0}+\frac{\zeta}{2}\right] \cup\left(\bigcup_{i=1}^{n}\left[\xi_{i}-\frac{\zeta}{2}, \xi_{i}+\frac{\zeta}{2}\right]\right) \cup\left[\xi_{n+1}-\frac{\zeta}{2}, \xi_{n+1}\right]\end{cases}
$$

and $0 \leq \phi(x) \leq 1$ for all $x \in[-1,1]$. It is obvious that

$$
\left|\Gamma_{k} y\right| \leq C\left\|y^{\left(n_{k}\right)}\right\|_{C\left(\bigcup_{i=1}^{n+1}\left[\xi_{i-1}+\zeta, \xi_{i}-\zeta\right]\right)} \leq C\left\|(\phi y)^{\left(n_{k}\right)}\right\|_{C[-1,1]} .
$$

By Theorem 3.10.4 in [15], for $y \in W_{q}^{\sigma}$, the estimate

$$
|\lambda|^{\sigma-n_{k}-q^{-1}}\left\|y^{\left(n_{k}\right)}\right\|_{C[-1,1]} \leq C\left(\|y\|_{q, \sigma}+|\lambda|^{\sigma}\|y\|_{q, 0}\right)
$$


holds. By Theorem 4.1, from (5.4), (5.5) it follows that, for all $\lambda \in \Omega_{\varepsilon}(\alpha, \beta)$ sufficiently large in modulus, the following estimates hold:

$$
\begin{aligned}
& |\lambda|^{\sigma-n_{k}-q^{-1}}\left|\Gamma_{k} y\right| \leq C|\lambda|^{\sigma-n_{k}-q^{-1}}\left\|(\phi y)^{\left(n_{k}\right)}\right\|_{C[-1,1]} \\
& \leq C\left(\|\phi y\|_{q, \sigma}+|\lambda|^{\sigma}\|\phi y\|_{q, 0}\right) \\
& \leq C(\varepsilon)\left(\left\|M_{0}(\lambda)(\phi y)\right\|_{q, \sigma-2}+|\lambda|^{\sigma-2}\left\|M_{0}(\lambda)(\phi y)\right\|_{q, 0}\right) \\
& \leq C(\varepsilon)\left(\left\|M_{0}(\lambda) y\right\|_{q, \sigma-2}+|\lambda|^{\sigma-2}\left\|M_{0}(\lambda) y\right\|_{q, 0}+\sum_{\ell=0}^{\sigma-1}|\lambda|^{\sigma-1-\ell}\|y\|_{q, \ell}\right) \\
& \leq C(\varepsilon)\left(\|M(\lambda) y\|_{q, \sigma-2}+|\lambda|^{\sigma-2}\|M(\lambda) y\|_{q, 0}+\|T y\|_{q, \sigma-2}\right. \\
& \left.+|\lambda|^{\sigma-2}\|T y\|_{q, 0}+\sum_{\ell=0}^{\sigma-1}|\lambda|^{\sigma-1-\ell}\|y\|_{q, \ell}\right) \\
& \leq C(\varepsilon)\left(\|f\|_{q, \sigma-2}+|\lambda|^{\sigma-2}\|f\|_{q, 0}+\|T y\|_{q, \sigma-2}\right. \\
& \left.+|\lambda|^{\sigma-2}\|T y\|_{q, 0}+\sum_{\ell=0}^{\sigma-1}|\lambda|^{\sigma-1-\ell}\|y\|_{q, \ell}\right) .
\end{aligned}
$$

By virtue of Theorem 1.7.7/2(b) and Remark 1.1.7/5 in [3], the following inequality

$$
\|y\|_{q, \ell} \leq \delta\|y\|_{q, \ell+1}+C(\delta)\|y\|_{q, 0}
$$

holds for any $\delta>0$. Then, by virtue of (5.7),

$$
\begin{aligned}
|\lambda|^{\sigma-n_{k}-q^{-1}}\left\|\Gamma_{k} y\right\| \leq & C(\varepsilon)\left(\|M(\lambda) y\|_{q, \sigma-2}+|\lambda|^{\sigma-2}\|M(\lambda) y\|_{q, 0}+\|T y\|_{q, \sigma-2}\right. \\
& \left.+|\lambda|^{\sigma-2}\|T y\|_{q, 0}+\sum_{\ell=0}^{\sigma-1}|\lambda|^{\sigma-1-\ell}\left(\delta\|y\|_{q, \ell+1}+C(\delta)\|y\|_{q, 0}\right)\right) \\
\leq & C(\varepsilon)\left(\|M(\lambda) y\|_{q, \sigma-2}+|\lambda|^{\sigma-2}\|M(\lambda) y\|_{q, 0}+\|T y\|_{q, \sigma-2}\right) \\
& +|\lambda|^{\sigma-2}\|T y\|_{q, 0}+C(\varepsilon)\left(\delta+C(\delta)|\lambda|^{-1}\right) \sum_{\ell=0}^{\sigma}|\lambda|^{\sigma-\ell}\|y\|_{q, \ell} .
\end{aligned}
$$

From conditions (2), (3), inequality (5.8), and Theorem 1.7.7/2 in [3], for any $\delta>0$, we have

$$
\begin{aligned}
& \|T y\|_{q, \sigma-2}+|\lambda|^{\sigma-2}\|T y\|_{q, 0}+\sum_{k=1}^{2(n+1)}|\lambda|^{\sigma-n_{k}-q^{-1}}\left(\left|\Gamma_{k} y\right|+\left|F_{k} y\right|\right) \\
& \leq C(\varepsilon)\left(\|M(\lambda) y\|_{q, \sigma-2}+|\lambda|^{\sigma-2}\|M(\lambda) y\|_{q, 0}\right)+\delta\left(\|y\|_{q, \sigma}+|\lambda|^{\sigma-2}\|y\|_{q, 2}\right) \\
& \quad+C(\delta)|\lambda|^{\sigma-2}\|y\|_{q, 0}+C(\varepsilon)\left(\delta+C(\delta)|\lambda|^{-1}\right) \sum_{\ell=0}^{\sigma}|\lambda|_{\sigma-\ell}\|y\|_{q, \ell} \\
& \quad+\sum_{k=1}^{2(n+1)}|\lambda|^{\sigma-n_{k}-q^{-1}}\|y\|_{q, n_{k}}
\end{aligned}
$$




$$
\begin{aligned}
\leq & C(\varepsilon)\left(\|f\|_{q, \sigma-2}+|\lambda|^{\sigma-2}\|f\|_{q, 0}\right) \\
& +C(\varepsilon)\left(\delta+C(\delta)|\lambda|^{-q^{-1}}\right) \sum_{\ell=0}^{\sigma}|\lambda|^{\sigma-\ell}\|y\|_{q, \ell} .
\end{aligned}
$$

Substituting (5.9) into (5.4) yields

$$
\begin{aligned}
\sum_{\ell=0}^{\sigma}|\lambda|^{\sigma-\ell}\|y\|_{q, \ell} \leq & C(\varepsilon)\left(\|f\|_{q, \sigma-2}+|\lambda|^{\sigma-2}\|f\|_{q, 0}+\sum_{k=1}^{2(n+1)}|\lambda|^{\sigma-n_{k}-q^{-1}}\left|f_{k}\right|\right. \\
& \left.+\left(\delta+C(\delta)|\lambda|^{-q^{-1}}\right) \sum_{\ell=0}^{\sigma}|\lambda|^{\sigma-\ell}\|y\|_{q, \ell}\right) .
\end{aligned}
$$

It is obvious that for fixed $\varepsilon>0$ it is possible to choose $\delta>0$ so small and $|\lambda|$ so large that $C(\varepsilon)\left(\delta+C(\delta)|\lambda|^{-q^{-1}}\right)<1$. Hence, for $\lambda \in \Omega_{\varepsilon}(\underline{w}, \bar{w})$ sufficiently large in modulus, we obtain a priori estimate (5.1).

It follows from estimate (5.1) that we can obtain the uniqueness property of solution of problem (1.1)-(1.2), i.e., the operator $\widehat{M}$ is a one-to-one operator. Moreover, by Theorem 3.1 the operator $\widehat{M}$ from $W_{q}^{\sigma}$ onto $W_{q}^{\sigma-2} \oplus \mathbb{C}^{2(n+1)}$ is Fredholm.

In view of condition (2), the operator $T$ from $W_{q}^{\sigma}$ into $W_{q}^{\sigma-2} \oplus \mathbb{C}^{2(n+1)}$ is compact. From above, we get that the operator $\widehat{M}$ is an isomorphism from $W_{q}^{\sigma}$ onto $W_{q}^{\sigma-2} \oplus \mathbb{C}^{2(n+1)}$. The proof is completed.

\section{Conclusion}

Sturm-Liouville problem with discontinuous points inside an interval has attracted extensive attention for its wide application in physical and mathematical fields. In this paper, we go into Sturm-Liouville problem with finite discontinuous points, and for such a problem, we establish the properties such as isomorphism, Fredholmness, and coerciveness with respect to the spectral parameter. These results are of both theoretical and practical significance.

\section{Acknowledgements}

Not applicable.

Funding

The work of the authors is supported by the National Nature Science Foundation of China (No. 11361039), the Inner Mongolia Natural Science Foundation (Nos. 2017MS0124, 2017MS0125, 2017MS(LH)0105), and the Inner Mongolia Autonomous Region University Scientific Research Project (Nos. NJZY17045, NJZC16165).

\section{Availability of data and materials}

Data sharing not applicable to this article as no datasets were generated or analysed during the current study.

\section{Competing interests}

The authors declare that there are no competing interests.

Authors' contributions

All authors contributed equally to the writing of this paper. The authors read and approved the final manuscript.

\section{Author details}

${ }^{1}$ School of Mathematical Sciences, Inner Mongolia University, Hohhot, P.R. China. ${ }^{2}$ College of Mathematics Science, Inner Mongolia Normal University, Hohhot, P.R. China. ${ }^{3}$ Inner Mongolia Agricultural University, Hohhot, P.R. China. ${ }^{4}$ School of Mathematical Sciences, Qufu Normal University, Qufu, P.R. China. 


\section{Publisher's Note}

Springer Nature remains neutral with regard to jurisdictional claims in published maps and institutional affiliations.

Received: 9 November 2018 Accepted: 16 January 2019 Published online: 23 January 2019

\section{References}

1. Likov, A.V., Mikhailov, Y.A.: The Theory of Heat and Mass Transfer. Qosenergaizdat, Moscow (1963) (Russian)

2. Agranovich, M.S.: Spectral properties of diffraction problems. In: Voitovich, N.N., Kazenelenbaum, B.Z., Sivov, A.N. (eds.) Generalized Method of Eigenoscillations in the Theory of Diffraction. Nauka, Moscow (1977)

3. Yakubov, S., Yakubov, Y.: Differential-Operator Equation Ordinary and Partial Differential Equation. Chapman \& Hall/CRC, Boca Raton (1999)

4. Shakhmurov, V.B.: Linear and nonlinear abstract elliptic equations with VMO coefficients and applications. Fixed Point Theory Appl. 2013, 6 (2013)

5. Rasulov, M.L.: Methods of Contour Integration. Nort-Holland, Amsterdam (1967)

6. Kandemir, M.: Irregular boundary value problems with discontinuous coefficients and the eigenvalue parameter. Mediterr. J. Math. 6, 317-338 (2009)

7. Kandemir, M., Yakubov, Y.: Regular boundary value problems with a discontinuous coefficient, functional-multipoint conditions, and a linear spectral parameter. Isr. J. Math. 180, 255-270 (2010)

8. Mukhtarov, O.S., Demir, H.: Coerciveness of the discontinuous initial-boundary value problem for parabolic equation. Isr. J. Math. 114, 239-252 (1999)

9. Mukhtarov, O.S., Yakubov, S.: Problems for ordinary differential equations with transmission conditions. Appl. Anal. 81 1033-1064 (2002)

10. Aydemir, K.: Boundary value problems with eigenvalue depending boundary and transmission conditions. Bound. Value Probl. 2014, 131 (2014)

11. Aydemir, K., Mukhtarov, O.S.: Second-order differential operators with interior singularity. Adv. Differ. Equ. 2015, 26 (2015)

12. Aydemir, K., Mukhtarov, O.S.: Spectrum and Green's function of a many-interval Sturm-Liouville problem. Z. Naturforsch. 70(5), 301-308 (2015)

13. Cai, J., Zheng, Z.: Inverse spectral problems for discontinuous Sturm-Liouville problems of Atkinson type. Appl. Math. Comput. 327, 22-34 (2018)

14. Cai, J., Zheng, Z: Matrix representations of Sturm-Liouville problems with coupled eigenparameter-dependent boundary conditions and transmission conditions. Math. Methods Appl. Sci. 41(9), 3495-3508 (2018)

15. Zheng, Z., Cai, J., Li, K., Zhang, M.: A discontinuous Sturm-Liouville problem with boundary conditions rationally dependent on the eigenparameter. Bound. Value Probl. 2018, 103 (2018)

16. Li, K., Sun, J., Hao, X.: Dependence of eigenvalues of 2 nth order boundary value transmission problems. Bound. Value Probl. 2017, $143(2017)$

17. Li, K., Sun, J., Hao, X.: Eigenvalues of regular fourth order Sturm-Liouville problems with transmission conditions. Math. Methods Appl. Sci. 40, 3538-3551 (2017)

18. Li, K., Sun, J., Hao, X., Bao, Q.: Spectral analysis for discontinuous non-self-adjoint singular Dirac operators with eigenparameter dependent boundary condition. J. Math. Anal. Appl. 453, 304-316 (2017)

19. Allahverdiev, B.P., Bairamov, E., Ugurlu, E.: Eigenparameter dependent Sturm-Liouville problems in boundary conditions with transmission conditions. J. Math. Anal. Appl. 401, 388-396 (2013)

20. Aliev, B.A.: Solvability of a boundary value problem for a second order elliptic-differential operator equation with spectral parameter in the equation and boundary conditions. Trans. NAS Azerb. 4, 3-16 (2010)

21. Margareth, S.A., Rivera, J.E., Mauricio, S., Villagran, O.V.: Transmission problem in thermoelasticity. Bound. Value Probl. 2011, Article ID 190548 (2011)

22. Cai, J., Zheng, Z:: A singular Sturm-Liouville problem with limit circle endpoints and eigenparameter dependent boundary conditions. Discrete Dyn. Nat. Soc. 2017(2), Article ID 9673846 (2017)

23. Buschmann, D., Stolz, G., Weidmann, J.: One-dimensional Schrödinger operators with local point interactions. J. Reine Angew. Math. 467, 169-186 (1995)

24. Kandemir, M., Mukhtarov, O.S.: Nonlocal Sturm-Liouville problems with integral terms in the boundary conditions. Electron. J. Differ. Equ. 2017, 11 (2017)

25. Kato, T.: Perturbation Theory for Linear Operators. Springer, Berlin (1966)

26. Besow, O.V., Il'in, V.P., Nikolskii, S.M.: Integral Representation of Functional and Embedding Theorems, vol. 1. State, New York (1978)

27. Dunford, N., Schwartz, J.T.: Linear Operators. Part II. Spectral Theory. Interscience, New York (1963)

28. Krein, S.G.: Linear Differential Equations in Banach Spaces. Am. Math. Soc., Providence (1971)

29. Krein, S.G.: Linear Equations in Banach Spaces. Birkhäuser, Basel (1982) 